\title{
Detecting Suspicious Motion with Nonimaging Sensors
}

\author{
Neil C. Rowe Ahren A. Reed, and Jose J. Flores \\ U.S. Naval Postgraduate School \\ Monterey, California, United States \\ ncrowe at nps.edu
}

\begin{abstract}
Automated distributed sentry systems need only detect suspicious behavior. Microphones and infrared detectors may suffice, as well as being simpler and cheaper than cameras while reducing invasion of privacy. We report on a new approach to analyzing data from acoustic sensors and binary infrared and magnetic sensors towards detecting changes in the velocity vectors of walking people. We show that changes in speed and direction can be detected directly by simple mathematical calculations from even imprecise sensing data. We show methods for detecting both locally and globally suspicious behavior. We present results of experiments supporting our approach, and discuss design of a wireless sensor network for this processing.
\end{abstract}

Keywords-sensors; suspiciousness; motion; acoustic; infrared; acceleration

\section{INTRODUCTION}

Detection of suspicious activity is a key part of sentry duties beyond detecting unauthorized people in an area. Suspicious behavior is not just anomalous, but shows additional characteristics of goal-changing and concealment [1], both of which can be detected through motion analysis. Automated sentry software that looks for suspicious behavior can be more reliable than that focusing solely on anomalies.

An important application is to key convoy routes in Iraq or Afghanistan that U.S. troops overuse due to lack of adequate alternatives. Adversaries can note the reuse and emplace improvised explosive devices along such routes with a higher degree of effectiveness [2, 3, 4]. Visual surveillance is difficult for this task. Most emplacing is at night, and infrared cameras are expensive when covering tens of kilometers of road. Cameras are hard to conceal, and suffer problems with occlusion (whether deliberate or accidental). Image processing can be unreliable with weak or distant signals, and privacy is more extensively invaded with camera surveillance.

A better solution could be to use inexpensive nonimaging sensors such as diffuse passive-infrared or acoustic sensors. Most suspicious behavior on a road can be recognized by nonimaging sensors in the right places since nonsuspicious users of a road usually traverse it with a constant speed. So a wireless sensor network need only report data rarely, alerting human security personnel as to where to focus their attention. This paper will examine the use of acoustic, passive-infrared, and magnetic sensors for this task.

\section{EXPLOITING FOOTSTEP AUDIO}

A simple way to detect motion of pedestrians is through their footsteps. Footstep periodicity helps distinguish them from many kinds of background noise. Microphones can be less expensive and more versatile than in-ground sensors [5, 6] which can suffer from effects of different propagation rates in ground materials.

We first discuss here how suspicious walking behavior can be detected by just one or two sensor nodes. We build on the insight from [7] that the clue of a significantly nonzero norm of the acceleration vector was responsible for $90 \%$ of the performance in detecting deliberately suspicious behavior in video, over other clues such as anomalousness of location, occlusion, and speed. Thus, a good way to detect suspicious behavior is to try to fit behavior to straight-line paths and flag the data that does not fit. This could be supplemented with classification of footsteps or gaits [8] to detect unusual ones.

We can use clues of the strength and time of the footsteps peaks to fit approaching and receding behavior. Acoustic signals generally follow an inverse square law in perceived strength with subject distance. We can also measure differences in the time of footsteps between two microphones to obtain additional constraints on subject position. Differences in time multiplied by the speed of sound give differences in distance, a measurable few milliseconds for most deployments.

\section{A. Detecting locally suspicious behavior using signal strengths}

Unlike seismic footstep signals which are filtered by the natural frequencies of the ground [5], audio footstep signals do not exhibit distinctive frequency patterns. They are easier to detect by examining overall sound energy after low-pass filtering. We first processed our 22khz audio by summing the absolute values for each 0.0045 seconds of the signal to get a compressed signal. We did a further smoothing with the filter $y_{i+1}=0.98 * y_{i}+0.02 * x_{i}$ to approximate background noise, and subtracted this from the compressed signal. This worked well at eliminating noise of traffic and wind which were common in our outdoors experiments. We discarded negative peaks since footsteps are always positive peaks, and low-pass filtered again to get a smoother waveform that was easier to analyze. Fig. 1 shows an example.

We then found the peaks of the waveform and measured their height, narrowness (ratio of average height before and after 0.045 seconds to the peak height), and asymmetry (ratio of the difference of the heights 0.045 seconds before and after to the peak height). (We also explored parameters from the Fourier transform, but they did not help performance.) 


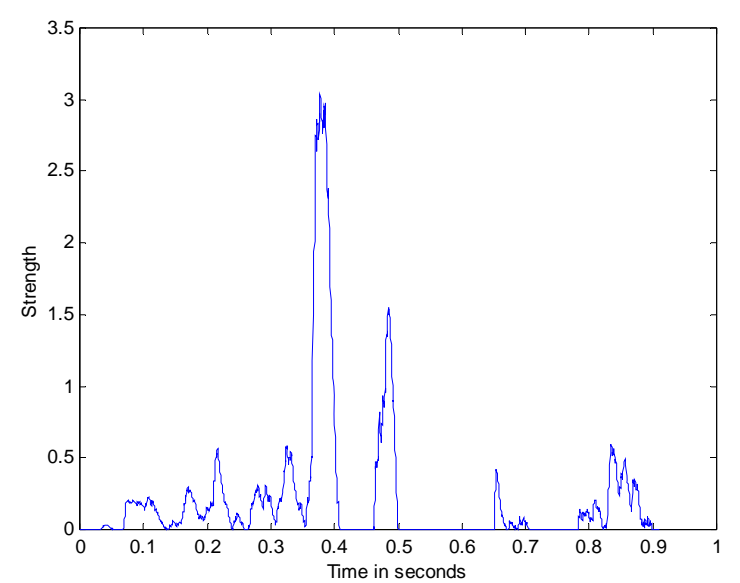

Figure 1. Example footsteps at a turn, followed by wind noise.

Following the literature summary in [5], normal footsteps of the same walker are not less than 0.48 seconds apart and no more than 0.80 seconds apart. We search for sequences of peaks that obey this constraint. The first moment improves its peak-time accuracy, and the second moment estimates "peak width" for an additional clue in matching. A useful additional constraint is that an object on a straight-line path with a constant speed shows no local minimum of the received signal, just a local maximum at its closest approach. Similarly, the time delay between a footstep and its detection should show only a local minimum for a single walker.

Consider three successive footsteps of pedestrian walking in a straight line at a constant speed, $\left(t_{1}, s_{1}\right),\left(t_{2}, s_{2}\right),\left(t_{3}, s_{3}\right)$. First assume that the generating sounds are of equal intensity, a good assumption for many instances of walking. Then $s_{i}=K_{0} /\left[D^{2}+v^{2}\left(t_{i}-t_{o}\right)^{2}\right]$ with unknowns $K_{0}$ (the signal loudness times the detection sensitivity), D (the distance of closest approach), $\mathrm{v}$ (the speed), and $t_{0}$ (the time of closest approach, or the projected time if the path was broken off before or after it got there). We can reduce this to three unknowns with $K=K_{0} / v$ and $t_{a}=D / v$ obtaining $s_{i}=K /\left[t_{a}{ }^{2}+\left(t_{i}-t_{o}\right)^{2}\right]$. Then with data from three footsteps, we can divide the equations to eliminate $\mathrm{K}$ :

$$
\begin{aligned}
& t_{a}{ }^{2}=-\left(s_{2}\left(t_{2}-t_{0}\right)^{2}-s_{1}\left(t_{1}-t_{0}\right)^{2}\right) /\left(s_{2}-s_{1}\right) \\
& t_{a}{ }^{2}=-\left(s_{3}\left(t_{3}-t_{0}\right)^{2}-s_{2}\left(t_{2}-t_{0}\right)^{2}\right) /\left(s_{3}-s_{2}\right)
\end{aligned}
$$

Equating them gives:

$$
\begin{gathered}
t_{0}=\left[\left(s_{2}-s_{1}\right)\left(s_{3} t_{3}{ }^{2}-s_{2} t_{2}{ }^{2}\right)-\left(s_{3}-s_{2}\right)\left(s_{2} t_{2}{ }^{2}-s_{1} t_{1}{ }^{2}\right)\right] \\
/ 2\left[\left(s_{2}-s_{1}\right)\left(s_{3} t_{3}-s_{2} t_{2}\right)-\left(s_{3}-s_{2}\right)\left(s_{2} t_{2}-s_{1} t_{1}\right)\right]
\end{gathered}
$$

Using $t_{0}$ we then calculate $t_{a}$ and $K=s_{1}\left[t_{a}{ }^{2}+\left(t_{1}-t_{o}\right)^{2}\right]$. Since these must be nonnegative along a path with zero acceleration, negative values represent either turns, multiple pedestrians, or (rarely) significant footstep intensity variation.

The equation for $t_{0}$ is most accurate when the signal strengths are quite different, and will be ill-conditioned if they are close (when it is hard to tell if a person is coming or going). We thus do not consider peak pairs whose heights are within $10 \%$ of one another. However, it is good to choose times close together because speed and direction are more consistent in shorter time periods.

Parameter $t_{a}=D / v$ is key because its changes along a path mean changes in either the distance of closest approach $\mathrm{D}$ (indicating a change of course) or the speed $\mathrm{v}$ (indicating slowing, speeding, or stopping), both of which can be suspicious. It is possible that no change in $t_{a}$ occurs on a course or speed change, as when the sensor lies near the perpendicular bisector of a path turn and the path speed is exactly constant, or if the path accelerates while it turns toward the sensor at the right speed. But these are rare coincidences.

We cluster $\left(t_{0}, t_{a}, K\right)$ estimates to find consistent paths for pedestrians. This idea has been used for distinguishing audio events besides footsteps [9]. Points that are not close to any cluster are either spurious or suspicious, and can be ignored. Distinct clusters represent different walkers, a walker stopping or slowing, a walker making a change in direction, or some combination.

\section{B. Handling variations in footstep loudness}

If footsteps vary significantly in loudness at their source, we can model this by differences in $\mathrm{K}$. A second sensor will be useful. Let $S_{i j}$ be the strength of the footstep sound i received by sensor $\mathrm{j}, t_{i j}$ the time that footstep sound $\mathrm{i}$ was received by sensor $\mathrm{j}, t_{0 j}$ the time of nearest approach to sensor $\mathrm{j}, t_{a j}=D_{0 j} / v$ the ratio of the distance of nearest approach to the velocity of the tracked object, and $K_{i}$ the loudness of footstep $i$ times the detectability of a sensor. Then at time $\mathrm{i}$ :

$$
K_{i}=s_{i 1}\left[t_{a 1}{ }^{2}+\left(t_{i 1}-t_{01}\right)^{2}\right]=s_{i 2}\left[t_{a 2}{ }^{2}+\left(t_{i 2}-t_{02}\right)^{2}\right]
$$

With good estimates of the $t_{0 j}$ values, we only need two times and signal strengths to solve for $t_{a 1}$ and $t_{a 2}$ since:

$$
\begin{aligned}
& t_{a 1}=\left[s_{i 2} s_{k 2}\left(t_{i 2}-t_{02}\right)^{2}-s_{i 1} s_{k 2}\left(t_{i 1}-t_{01}\right)^{2}\right. \\
& \left.+s_{i 2} s_{k 1}\left(t_{k 1}-t_{01}\right)^{2}-s_{i 2} s_{k 2}\left(t_{k 2}-t_{02}\right)^{2}\right] \\
& /\left(s_{i 1} s_{k 2}-s_{i 2} s_{k 1}\right) \\
& t_{a 2}=\left(s_{i 1} / s_{i 2}\right)\left[r_{1}+\left(t_{i 1}-t_{01}\right)^{2}\right]-\left(t_{i 2}-t_{02}\right)^{2}
\end{aligned}
$$

A rough approximation of $t_{0 j}$ is the time of the highest peak at sensor $\mathrm{j}$. We can do better with a three-point 
quadratic approximation $s_{i j}=a_{j} t_{i j}{ }^{2}+b_{j} t_{i j}+c$ for which the peak is at $t_{0 j}=-b_{j} / 2 a_{j}$. With three points for sensor

j:

$$
a_{j}=\left[\left(s_{1 j}-s_{2 j}\right) /\left(t_{1 j}-t_{2 j}\right)\left(t_{1 j}-t_{3 j}\right)\right]
$$

$$
-\left[\left(s_{2 j}-s_{3 j}\right) /\left(t_{2 j}-t_{3 j}\right)\left(t_{1 j}-t_{3 j}\right)\right]
$$

$b_{j}=\left[\left(s_{1 j}-s_{2 j}\right) /\left(t_{1 j}-t_{2 j}\right)\right]-a\left(t_{1 j}+t_{2 j}\right)$.

To match peaks between two sensors, we must first synchronize them. We iterate over possible reasonable time shifts to find the one that permits the maximum number of matches within a threshold.

Results on experiments showed around a $60 \%$ accuracy in detecting deliberate stops and turns along the path indoors, and $30 \%$ outdoors. The main problem was acoustic noise.

\section{Using time delay as well as loudness}

Another feature of a footstep that we can exploit is the differences in its time of arrival at two or more sensors. Footsteps are too irregular in frequencies to provide Doppler shifts. But if we can compare time gaps between different sensors, we can estimate the difference in distances to the sensors. Suppose the distances to the source at some instant of time are $d_{1}$ and $d_{2}$, w is the amount of time later that the peak appears at sensor 2, and $\mathrm{c}$ is the speed of sound. Then $s_{1} d_{1}{ }^{2}=s_{2} d_{2}{ }^{2}$ and $c w=d_{2}-d_{1}$, whose only positive solution is $d_{1}=c w\left[s_{2}+\sqrt{s_{1} s_{2}}\right] /\left(s_{1}-s_{2}\right) \quad$ and $d_{2}=c w\left[s_{1}+\sqrt{s_{1} s_{2}}\right] /\left(s_{1}-s_{2}\right)$. In a Cartesian coordinate system with the sensors at $(0,0)$ and $\left(d_{12}, 0\right)$, we know three sides of a triangle, and the source must be at $\left(\left(d_{1}^{2}-d_{2}^{2}+d_{12}^{2}\right) / 2 d_{12}, \sqrt{d_{1}^{2}-x^{2}}\right)$. We can then calculate three successive locations of the pedestrian, and flag as suspicious any path of significantly nonzero acceleration. This gives us an alternative to using signal strengths alone, and it may be more accurate. However, it does require that we accurately measure peaks within milliseconds, which we could not do for our experiments.

\section{Globally suspicious behavior}

Behavior can also be suspicious in wider time windows. For instance, a sequence of footsteps that suddenly terminate, at an apparent distance that should be well within sensor range, is suspicious, as are footsteps that change their average rate of increase or decrease (indicating a change in direction). We term this "global" suspicion as opposed to the "local" above. Global clues can supplement local clues in assessment of many kinds of deception [10].

For such analysis we first extract likely sequences of footsteps. Again assuming gaps of 0.48-0.80 seconds between footsteps, we use dynamic programming where we first compute possible two-step sequences, then three-step sequences, and so on. We use the evenness of the gaps in a sequence as a way to rate sequences, and keep only the best- rated sequences among those with the same starting and ending peaks. We then rate the peaks by the length of their longest sequence divided by $1+2 \mathrm{D}$ where $\mathrm{D}$ is the average time-gap unevenness (time prediction error) for the sequence. For example for Fig. 1, the footsteps on the left are rated an average of 4.0 while the noise after time 0.6 is rated 2.0. This analysis gave us an average in footstep detection of $72 \%$ recall with $74 \%$ precision indoors, and $94 \%$ recall with $47 \%$ precision outdoors, a significant improvement over detection with local measurements. This approach also allows us to distinguish footsteps of multiple pedestrians.

Then a quick measure of global suspiciousness is the degree to which the global maximum is at the center of the sequence. For instance, the uneven footstep distribution in Fig. 1 is suspicious. It represents a right-angle turn by the subject in front of the microphone, after which the sound decreases quickly.

\section{E. Experimental details}

We used inexpensive cardioid microphones with Icicle preamplifiers plugged into a computer running the Windows "Sound Recorder" software. Microphones were lain on the ground in the direction of the expected footsteps; the cardioid response was helpful in narrowing the response focus. When we used two microphones, one pointed at the other at a distance to reduce effects of the subject azimuth angle.

The response pattern of microphones was determined by using a fixed-signal clicking device at 50 locations at 8 evenly spaced bearings in one foot increments from the microphone tip. We found that a good model was $H=\left(K_{1}+K_{2} \cos ^{2}(\theta-B)\right) /\left(D+K_{3}\right)^{2} \quad$ for received signal strength where $\theta$ is the source bearing, B the microphone orientation, and $\mathrm{D}$ the distance to the source. Indoors on carpet the constants were $(5.4,1.2,6.7)$, and outdoors on pavement, $(15.0,10.0,6.7)$.

We did experiments with a range of behavior and footwear. To simulate our target environment of roads, we used an outdoors configuration 100 feet long and 20 feet wide. Indoors experiments focused on straight-line tracks: from near one microphone to near the other, along the perpendicular bisector of the microphone locations, and at a 45 degree angle to the line connecting the microphones through the midpoint.

\section{EXPLOITING INFRARED AND MAGNETIC SENSORS}

An alternative for detection of suspicious behavior is passive-infrared or magnetic sensing. Change-detecting sensors are common in automated surveillance tasks, and they can use several modalities. They can be inexpensive and have low power consumption because messages need not be transmitted unless evidence of change is strong. A weakness of such sensors is that wind, lighting conditions, background electronic noise, and routine traffic can generate false alarms. To reduce the false alarms, we must track changes over a period of time to find consistent patterns. 
We explored Crossbow MSP410 motes for detection of suspicious motion. They contain passive-infrared (PIR) and magnetic change-detecting sensors. [11] uses a similar approach with some different assumptions. Improvements to this kind of technology are actively being explored [12] but are still expensive.

\section{A. Exploitation of change-detection information}

Our previous work [7] explored the magnetic sensors in these motes, so the current work focused on the moreversatile passive-infrared sensors. These are binary motion sensors (they either detect motion or do not). Each mote has sensors for four azimuth quadrants, and more than one quadrant can signal detection for objects between quadrants.

Signal strengths are sent by the mote but are not very helpful. Background PIR levels generally range 580 to 690 both indoors and out. Most readings when motion is detected are the maximum reading of 1023, though sometimes the mote gives very low readings. After study, we concluded that any message specifying a quadrant indicates motion detection regardless of the reported strength. Signals of high strength but no quadrant occurred when a subject was standing still within the range of a mote, and suggest detection of body heat, but this detection is not reliable.

We found that moving people could be reliably detected to about 12 feet of distance, after which reliability deteriorated. Usually people were detected at their closest approach to the sensor, when their infrared signature was usually changing the most quickly. From experiments under a range of conditions, we concluded that the motes were close to $100 \%$ accurate in detecting approaches within 12 feet, and then exhibited a linear decrease to zero in their probability of detection up to 24 feet. This gives some clues to subject location. If a mote signals detection in a quadrant, and we have no prior probabilities of its location, we can assume that it is at the centroid of its probability distribution for an initial approximation. The mean radius for a circular sector subtending angle $\theta$ is $\int_{0}^{R_{1}} r * 2 \pi r \theta d r / \pi r^{2} \theta=2 R_{1} / 3$, and the mean radius in the annulus for weighting by the inverse of distance is $\int_{R_{1}}^{R_{2}} r^{*}(2 \pi r / r) \theta d r / \pi\left(R_{2}{ }^{2}-R_{1}^{2}\right) \theta=\left(R_{1}+R_{2}\right) / 2$. If total probability of an object being in the sector is proportional to its area of $\pi R_{1}^{2} \theta$, the total probability of an object being in the annulus is proportional to half its area of $\pi\left(R_{2}{ }^{2}-R_{1}{ }^{2}\right) \theta$ since probability decreases linearly from 1 to 0 with radius. Therefore the probability of a detected object being in the sector is $p_{s}=2 R_{1}^{2} /\left(R_{1}^{2}+R_{2}^{2}\right)$. So the expected mean radius when objects can be in either the sector or the annulus is $\left[p_{s} * 2 R_{1} / 3\right]+\left[\left(1-p_{s}\right) *\left(R_{1}+R_{2}\right) / 2\right]$. For our experimental observations of $R_{1}$ of 12 feet and $R_{2}$ of 24 feet, this is 12.8 feet. We can also estimate that a detection in a quadrant is at the bearing of the center of the quadrant.

The motes also report significant magnetic changes due to nearby transit of ferromagnetic materials. Our subjects had keys in their pockets, and some experiments were in buildings where people were working, so we did get some magnetic reports. Since these messages are nondirectional, we interpret them as estimating a position centered on the mote.

\section{B. $\quad$ Fitting path segments with binary sensors}

To again apply the insight that suspicious behavior is correlated with nonzero acceleration vectors, we fit the estimated locations of objects detected by infrared and magnetic sensors to a straight space-time line defined by $x=x_{0}+v_{x} t, y=y_{0}+v_{y} t$. When subjects significantly deviate from this line, they must have a significant acceleration. An alternative is careful tracking using optimization techniques such as the Viterbi algorithm [13], then analyzing the tracks.

Given estimated subject positions $\left(x_{s i}, y_{s i}, t_{s i}\right)$, we do least-squares fitting on the estimated points with error $S=\sum_{i=1}^{N}\left[\left(x_{0}+v_{x} t-x_{i}\right)^{2}+\left(y_{0}+v_{y} t-y_{i}\right)^{2}\right]$. Optimal values for the unknowns $x_{0}, y_{0}, v_{x}$, and $v_{y}$ are:

$$
\begin{aligned}
& v_{x}=(\overline{x t}-\bar{x} * \bar{t}) /\left(\overline{t^{2}}-(\bar{t})^{2}\right), x_{0}=\bar{x}-v_{x} \bar{t} \\
& v_{y}=(\overline{y t}-\bar{y} * \bar{t}) /\left(\overline{t^{2}}-(\bar{t})^{2}\right), y_{0}=\bar{y}-v_{y} \bar{t}
\end{aligned}
$$

We calculate this fit for a sequence of points, then recursively split the sequence into segments whose fit is within a threshold. We found a threshold of 5 feet on the absolute error worked well with this data. However, our data involved multiple subjects sometimes walking independently. So we used a first phase that clusters points likely to belong to the same subject. It maintains a set of clusters and assigns points in time order to the cluster nearest in space-time distance of $D=\sqrt{\left(x-x_{c}\right)^{2}+\left(y-y_{c}\right)^{2}+\left(0.2 *\left(t-t_{c}\right)\right)^{2}} \quad$ where $(x, y, t)$ is the point and $\left(x_{c}, y_{c}, t_{c}\right)$ is the most recent point of the cluster, where $x$ and $y$ are measured in feet and $t$ in seconds. The 0.2 is because walking subjects average about five feet per second. If no cluster can be found within a distance threshold of 15, a new subject is assumed and a new cluster created. Once clusters have been created, we recursively split clusters until their fit is sufficient.

\section{Reasoning about probability distributions of location}

To improve position estimates still further, we create and update probability distributions of location in space and time [14]. This simplifies data fusion of from different sensors and different sensing modalities. In our experiments, we 
used a one-foot resolution for the probability distribution and a timestep of one second. The quadrant-specific infrared data provides probabilities within a 90-degree sector to a radius of 24 feet. Quadrantless infrared data provides weak evidence within a radius of 30 feet. Magnetic data provides evidence within a radius of 20 feet. Acoustic data provides peaks that give a crescent-shaped distribution extending to 50 feet, but is less reliable than the other sensors due to ambient noise.

Probabilities at the same location and timestep are added, since they are small and this approximates a disjunctive combination of independent probabilities. The distribution at the previous timestep is also added to these probabilities but with a "decay" and a "blur" whereby some of a probability is distributed to its neighbors to model possible movement.

We then search the probability distribution at each timestep to find local maxima above a threshold. We compute the centroid of the part of the distribution closest to each maximum and interpret these centroids as subject locations. We then fit the points using the methods of III.B. This method is robust to missing sensor reports because peaks at previous timesteps will remain albeit decayed. Note that spurious peaks will tend not to fit into the larger clusters and will be ignored.

\section{Experiments}

We conducted experiments with sensors arranged in various grid configurations to determine their capabilities. In the final summary experiments, we arranged 16 sensors in a 4 by 4 square grid with 5 feet between each sensor in a parking lot. Three subjects then walked across the sensor grid in various patterns. Six experiments were conducted: (70) all three subjects cross the field together in a straight line with constant speed; (71) all three subjects cross the field in a straight line but loiter in the center for a short time; (72) like the last but only one subject stops; (73) all three subjects enter the field and make a left turn of 90 degrees in the center of the field; (74) like the last but only one subject makes the turn while the others continue straight; and (75) two subjects enter the field from opposite sides and another crosses at right angles to them, all at constant speed.

Fig. 2 shows results from Experiment 73 plotted in three dimensions with time as the vertical axis, using only infrared and magnetic data. Path segment clusters are shown in different colors, with gray circles on the bottom indicating the locations of the infrared/magnetic sensors. In the coordinate system, the 16 sensors were at locations whose latitude and longitude coordinates were drawn from the set $\{0,18,36,54\}$ measured in feet.

Fig. 2 shows that preliminary setup in the center of the field and finalizing actions on the left side of the field were picked up properly. Fig. 2 shows a significant improvement in the quality of the estimates on those using audio alone. In general, partitioning of paths at stopping and turns was excellent with our software, because such events tend not to trigger many sensor events during the stop and turn,

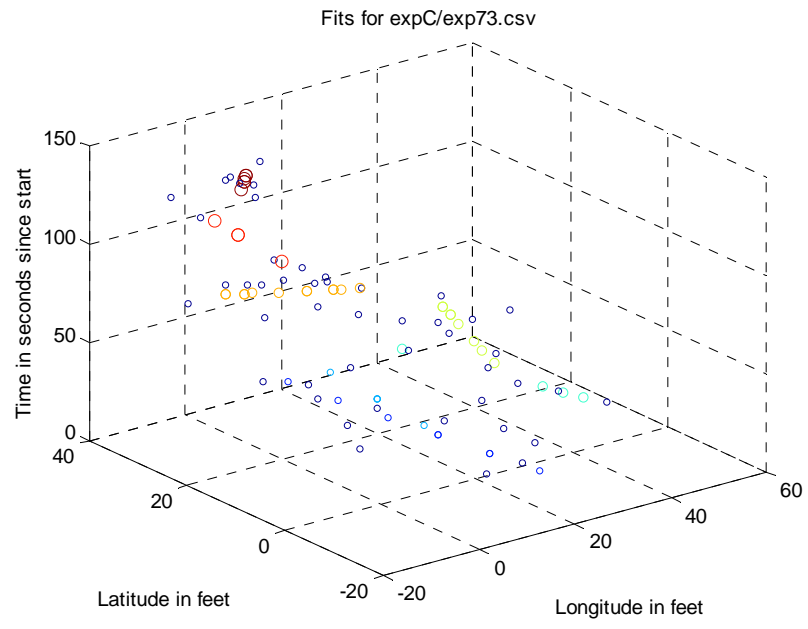

Figure 2. Clustering in space-time of estimated locations for Experiment 73 with setup and post-experiment activities.

simplifying the partitioning. Thus, our approach seems promising for detecting candidate suspicious behavior.

\section{E. Data fusion of audio in the experiments}

We also recorded audio from a single microphone during these experiments. The microphone was lain on the ground at $(37,37)$ in a northeast direction. Since the outdoor environment was noisy, we used only the global analysis method of II.D, and rated peaks by the length of their sequence. For each peak, we created a "crescent" probability distribution by normalizing the likelihoods $\sqrt{\min \left(S_{i}, H_{i}\right) / \max \left(S_{i}, H_{i}\right)}$ where $S_{i}$ is measured audio peak height and $H_{i}=\left(K_{1}+K_{2} \cos ^{2}\left(\theta_{i}-B\right)\right) /\left(D_{i}+K_{3}\right)^{2}$ from II.E. Fig. 3 shows an example probability distribution of the positions of subjects in Experiment 70 after the subjects passed the microphone and generated both infrared (top) and audio (bottom) data, each with their patterns. Brightness indicates increased probability.

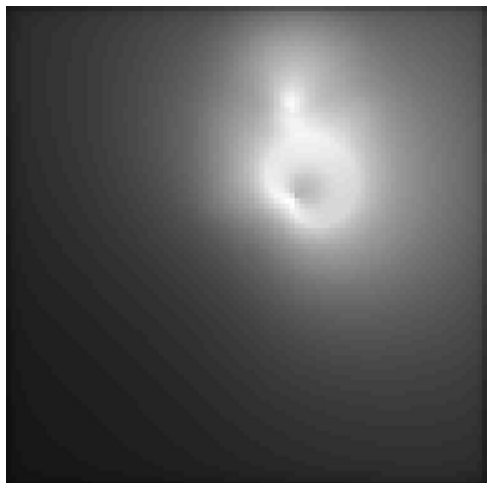

Figure 3. Example probability distribution from infrared and audio footstep detection. 
Inclusion of data from one microphone had limited benefit for these experiments because it was quite localized and many audio peaks that it found were spurious. But it gave helpful data when subjects were nearby. In Experiment 75, for instance, it gave us 102 additional events to supplement the 83 infrared and magnetic events. Welldistributed microphones should provide a different kind of probability distribution that should resolve many ambiguities.

\section{F. Finding suspicious behavior from path segments}

To use our segment fits for suspiciousness analysis, we can focus on their ends. If these are not on the edge of the sensor field, they represent potentially suspicious activity: either time gaps, changes in direction, or changes in speed, all of which will result in discontinuous segments with our recursive fitting. In Experiments 70-75, we were successful in finding suspicious behavior of groups of people but not all that of individuals. Usually it is clear which segments belong to the same person because people are limited in speed; thus we can rate suspiciousness at segment gaps by the degree of discontinuity. Note that negative evidence for suspiciousness is unnecessary, unlike with acoustic data, since all paths that stop must start again eventually to leave the sensor field.

\section{BUILDING A NETWORK OF SUSPICIOUS-BEHAVIOR SENSORS}

Our approach can support a wireless network of "suspicious-behavior" sensors. Along roads the sensors can be arranged in a line. Acoustic sensors with the simple microphone-preamplifier combinations we used could be located at 30 feet apart along the road to obtain adequate coverage. They need only communicate with a single neighbor sensor along a path to detect suspicious behavior using the methods of sections II.B or II.C. Conclusions of suspicious behavior could be forwarded along the road to occasional motes with larger antennas for transmitting reports to a base station [14]. Messages reported by the larger antennas should give the locations, times, and degree for the occasional suspicious events. One design would be to have 100 acoustic sensors per mile along roads, with 9 out of 10 having small antennas for neighbor transmission, and 1 out of 10 having larger antennas for transmission to a base station.

Passive-infrared sensors and acoustic sensors using our the methods have more need to pool their information to detect suspicious behavior, but not too much. Normal walking people will deviate over time an increasing amount from a linear space-time fit as described in section III.B, so attempts at fits for more than 10 seconds will be less successful. This suggests that 10 feet apart would be better because they would average one sensor report every two seconds. It would be desirable for motes to share the larger antenna for all modalities. Reliability could be improved if each mote redundantly sends messages in both directions along the road, which will reduce the probability of failure to roughly its square. Note that the base station can exclude sensor reports that are inconsistent due to sensor or transmission errors. The base station can also transmit orders to sensors to report additional information about emerging patterns. If a sufficient suspicious behavior is reported in an area, the base station can always alert human security personnel to inspect it themselves.

\section{CONCLUSIONS}

We have shown that nonimaging sensors have promise as a new way to detect suspicious behavior. But we need to conduct more experiments on a larger scale and over a longer time period to provide a better test of our methods.

\section{ACKNOWLEDGMENT}

This work was supported by the U.S. National Science Foundation under grant 0729696 of the EXP Program.

\section{REFERENCES}

[1] N. Rowe, "Detecting Suspicious Behavior from Only Positional Data with Distributed Sensor Networks," $\quad$ Proc. $5^{\text {th }}$ International Conference on Multibody Systems, Nonlinear Dynamics and Control, Long Beach, California, September 2005.

[2] R. Atkinson, "Left of boom," Washington Post, September 29 October 3, 2007.

[3] G. Grant, “Insurgency chess match,” DefenseNews, February 27, 2006.

[4] T. Magness, "IED defeat: observations from the National Training Center," Engineer (U.S. Army), pp. 28-31, January-March 2005.

[5] J. Sabatier and A. Ekimov, "A Review of Human Signatures in Urban Environments Using Seismic and Acoustic Methods," Proc. IEEE Conf. on Technologies for Homeland Security, pp. 215-220, May 2008.

[6] G. de Silva, T., Yamasaki, and K. Aizawa, "Evaluation of Video Summarization for a Large Number of Cameras in Ubiquitous Home," Proc. 13th annual ACM Intl. Conf. on Multimedia, Singapore, pp. 820-828, 2005.

[7] N. Rowe, M. O'Hara, and G. Singh, "Wireless Sensor Networks for Detection of IED Emplacement," Intl. Command and Control Research and Technology Symposium, Washington, DC, June 2009.

[8] A. Itai and H. Yasukawa, "Footstep Classification Using Simple Speech Recognition Technique," Proc. Intl. Symp. on Circuits and Systems, Seattle, WA, pp. 3234-3237, May 2008.

[9] R. Radhakrishnan, A. Divakaran, and P. Smaragdis, "Systematic Acquisition of Audio Classes for Elevator Surveillance," Mitsubishi Electric Research Laboratories, Technical Report TR2005-076, December 2005.

[10] A. Vrij, Detecting Lies and Deceit: The Psychology of Lying and the Implications for Professional Practice. Chichester, UK: Wiley, 2000.

[11] B. Song, H. Choi, and H. Lee, "Surveillance Tracking System Using Passive Infrared Motion Sensors in Wireless Sensor Network.," Proc. Intl. Conf. on Information Networking, Busan, China, January 2008, pp. 1-5.

[12] P. Zappi, E. Farella, and L. Benini, "Enhancing the Spatial Resolution of Presence Detection in a PIR Based Wireless Surveillance Network.," Proc. IEEE Conf. on Advanced Video and Signal Based Surveillance, London, UK, September 2007, pp. 295300 .

[13] S. Oh and S. Sastry, "Tracking on a Graph.," Proc. 4th Intl. Symp. on Sensor Networks, Los Angeles, USA, 2005, pp. 195-202.

[14] Zhao, F., and Guibas, L., Wireless Sensor Networks: An Information Processing Approach. San Francisco: Morgan Kaufmann, 2004. 
This paper appeared in the Third IEEE International Workshop on Bio and Intelligent Computing, Perth Australia, April 2010. 